\title{
Gd-EOB-DTPA-enhanced and diffusion-weighted magnetic resonance findings in hepatic epithelioid angiomyolipoma: A case report
}

\author{
JUN SUN, SHOUAN WANG, WENXIN CHEN and JINGTAO WU
}

Department of Radiology, Northern Jiangsu People's Hospital, Yangzhou University, Yangzhou, Jiangsu 225001, P.R. China

Received September 29, 2014; Accepted May 14, 2015

DOI: $10.3892 / 01.2015 .3368$

\begin{abstract}
The present study reports a case of histologically proven hepatic epithelioid angiomyolipoma that was evaluated with gadolinium ethoxybenzyl diethylenetriamine pentaacetic acid (Gd-EOB-DTPA)-enhanced magnetic resonance imaging and diffusion-weighted imaging. A 23 -year-old female was admitted to the Northern Jiangsu People's Hospital (Yangzhou, Jiangsu, China) due to a 5.6- $\mathrm{cm}$ mass in the liver, and a right partial hepatectomy was performed. Magnetic resonance imaging (MRI) revealed a hypointense mass on T1-weighted imaging, and a hyperintense mass on T2-weighted and diffusion-weighted imaging, with a higher apparent diffusion coefficient value compared with normal liver parenchyma. On the dynamic Gd-EOB-DTPA-enhanced MRI scan, the lesion manifested as hypervascular with multiple filiform vessels and a pseudocapsule image, and in the hepatobiliary phase the lesion demonstrated a lack of contrast retention, thus appearing hypointense compared with the background liver. Pre-operatively, EMAL was diagnosed on the basis of these findings in the tumor. The optimum treatment is complete surgical excision and subsequent follow-up. The patient was healthy and free from recurrence at 6 months and 1 year post-surgery. Therefore, knowledge of EAML specific features on dynamic Gd-EOB-DTPA-enhanced and DWI scans may improve the diagnostic accuracy of hypervascular hepatic tumors and may facilitate treatment selection.
\end{abstract}

\section{Introduction}

Hepatic epithelioid angiomyolipoma (EAML), a recently recognized subtype of AML, is very rare. At present, only sporadic cases have been reported. It is difficult to diagnose

Correspondence to: Mr. Jingtao Wu, Department of Radiology, Northern Jiangsu People's Hospital, Yangzhou University, 98 Nantong West Road, Yangzhou, Jiangsu 225001, P.R. China E-mail: drwujingtao@126.com

Key words: liver, epithelioid angiomyolipoma, magnetic resonance imaging, magnetic resonance contrast agents, diffusion-weighted imaging hepatic EAML prior to surgery, as there are no specific clinical, laboratory or radiological signs of the disease $(1,2)$. Recently, the use of gadolinium ethoxybenzyl diethylenetriamine pentaacetic acid (Gd-EOB-DTPA) as a liver-specific magnetic resonance (MR) contrast agent has been shown to be useful for improving the detection and characterization of hepatic lesions (3-5). In addition, diffusion-weighted (DW) MR imaging (MRI) is increasingly being applied as a liver imaging technique to differentiate between benign and malignant diseases by measuring the value of the apparent diffusion coefficient (ADC) based on extracellular water diffusion $(6,7)$. Although several case studies have reported imaging findings of hepatic EMAL with ultrasonography, computed tomography and MRI (1), to the best of our knowledge, this is the first study to describe the imaging findings of hepatic EMAL using Gd-EOB-DTPA-enhanced and DW MRI. In addition, the study compared the imaging and pathological findings of the disease. Written informed consent was obtained from the patient for publication of this study.

\section{Case report}

On February 20, 2012, a 23-year-old female experienced discomfort in the right upper quadrant of the abdomen for 5 days prior to hospitalization in the Northern Jiangsu People's Hospital (Yangzhou, Jiangsu, China). The physical examination indicated that the abdomen was soft, but not tender. The lower margin of the liver could be detected at approximately three-fingers distance from the right subcostal region, and there was no percussion pain around the hepatic area. The patient had no medical history of hepatitis and infectious diseases, such as schistosomiasis, and the patient did not use oral contraceptives. The results of serum examination showed an $\alpha$-fetoprotein (AFP) level of $1.92 \mathrm{ng} / \mathrm{ml}$ (normal range, $0-7 \mathrm{ng} / \mathrm{ml}$ ) and a carcinoembryonic antigen level of $1.66 \mathrm{ng} / \mathrm{ml}$ (normal range, $0-5 \mathrm{ng} / \mathrm{ml}$ ).

MRI of the upper abdomen was also performed using a 3.0-Tesla MR Scanner (Signa HDx; GE Medical Systems, Milwaukee, WI, USA). Axial T1-weighted imaging (WI), T2WI (fat saturation), DWI (b value, 0; $500 \mathrm{sec} / \mathrm{mm}^{2}$ ), and liver acquisition with volume acceleration (dynamic contrast enhancement) were used after Gd-EOB-DTPA (Primovist, Bayer-Schering, Berlin, Germany) was injected manually at $0.025 \mathrm{mmol} / \mathrm{kg}$ body weight. 
A

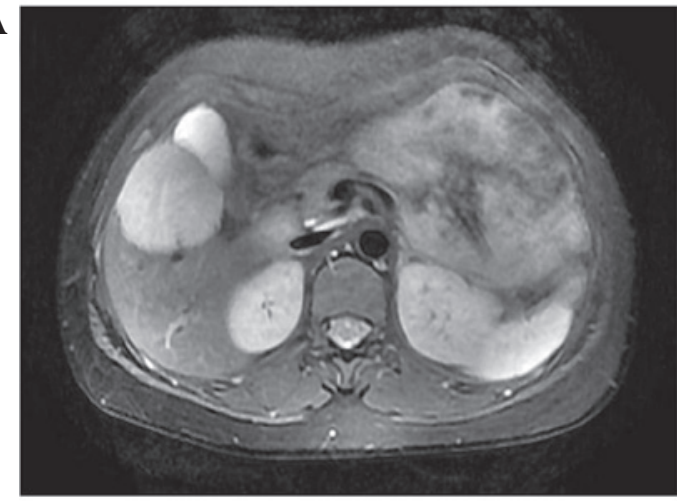

B

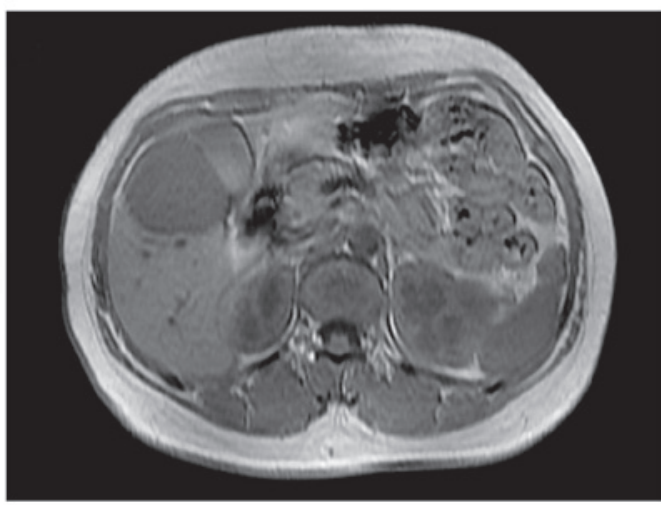

Figure 1. (A) Axial T2-weighted imaging with fat saturation showing a heterogeneous hyperintense mass in segment 5 of the right hepatic lobe. (B) T1-weighted image showing the lesion with a homogeneous low signal intensity.

A

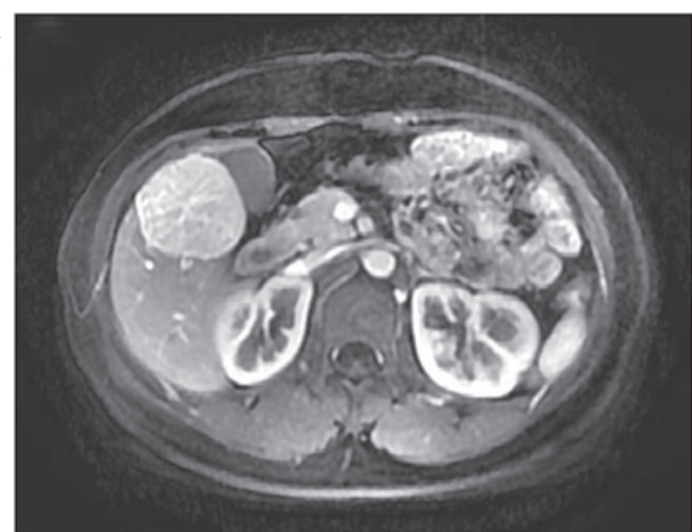

$\mathbf{C}$

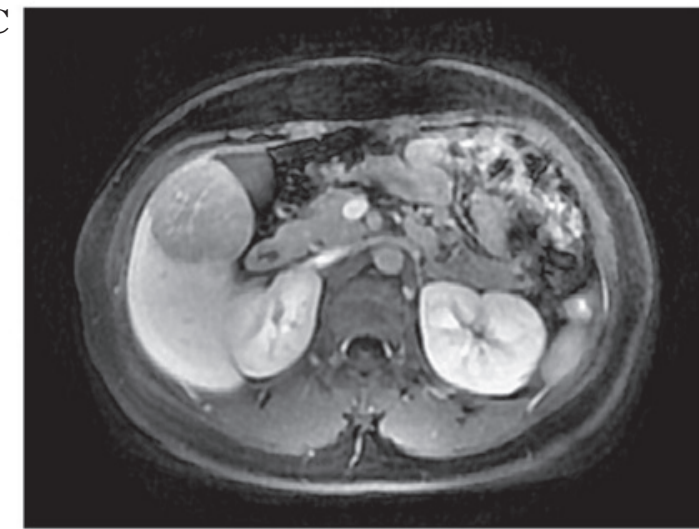

B

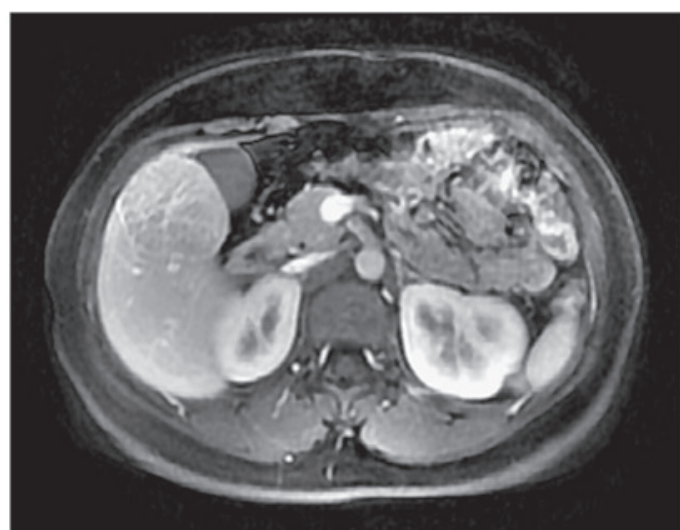

D

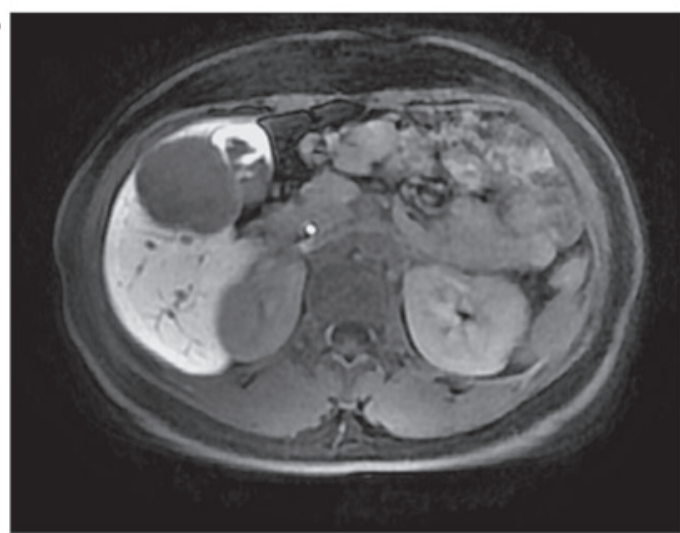

Figure 2. (A) Markedly heterogeneous high signal intensity in the arterial phase of the dynamic gadolinium ethoxybenzyl diethylenetriamine pentaacetic acid (Gd-EOB-DTPA)-enhanced scan, with multiple central filiform vessels and capsule enhancement. (B) The signal intensity was relatively reduced in the portal venous phase, but slightly higher than the surrounding liver parenchyma, with an enhanced vascular signal visible in the lesion. (C) The signal intensity of the tumor was lower at the parenchymal phase compared with the surrounding liver parenchyma. (D) A lack of Gd-EOB-DTPA uptake was noted in the hepatobiliary phase at 30 min post-injection.

An MRI scan of the abdomen showed an $\sim 5.6-\mathrm{cm}$, well-defined, round tumor in segment 5 of the right hepatic lobe. The tumor showed a heterogeneous high signal intensity on T2WI (Fig. 1A) and a homogeneous low signal intensity on T1WI (Fig. 1B). Gd-EOB-DTPA-enhanced MRI showed markedly heterogeneous enhancement in the arterial phase, and central filiform vessels and capsule enhancement were also observed (Fig. 2A). The signal intensity of the lesion was relatively reduced in the portal venous phase, but remained slightly higher than the surrounding liver parenchyma (Fig. 2B), and the enhanced signal intensity of the vascular image was visualized within the tumor. The signal intensity of the lesion was lower than the surrounding liver parenchyma in the delay phase (Fig. 2C). The signal intensity of the tumor was relatively homogeneous, but markedly lower compared with that of the liver parenchyma, as a defect in the hepatobiliary phase (Fig. 2D). Moreover, a high signal intensity on DWI (b value, $500 \mathrm{sec} / \mathrm{mm}^{2}$ ) was obtained for hepatic EAML (Fig. 3A), with an average ADC value of $1.99 \times 10^{-3} \mathrm{~mm}^{2} / \mathrm{sec}$, which was higher than that of the normal liver parenchyma $\left(1.45 \times 10^{-3} \mathrm{~mm}^{2} / \mathrm{sec}\right)($ Fig. $3 \mathrm{~B})$. 

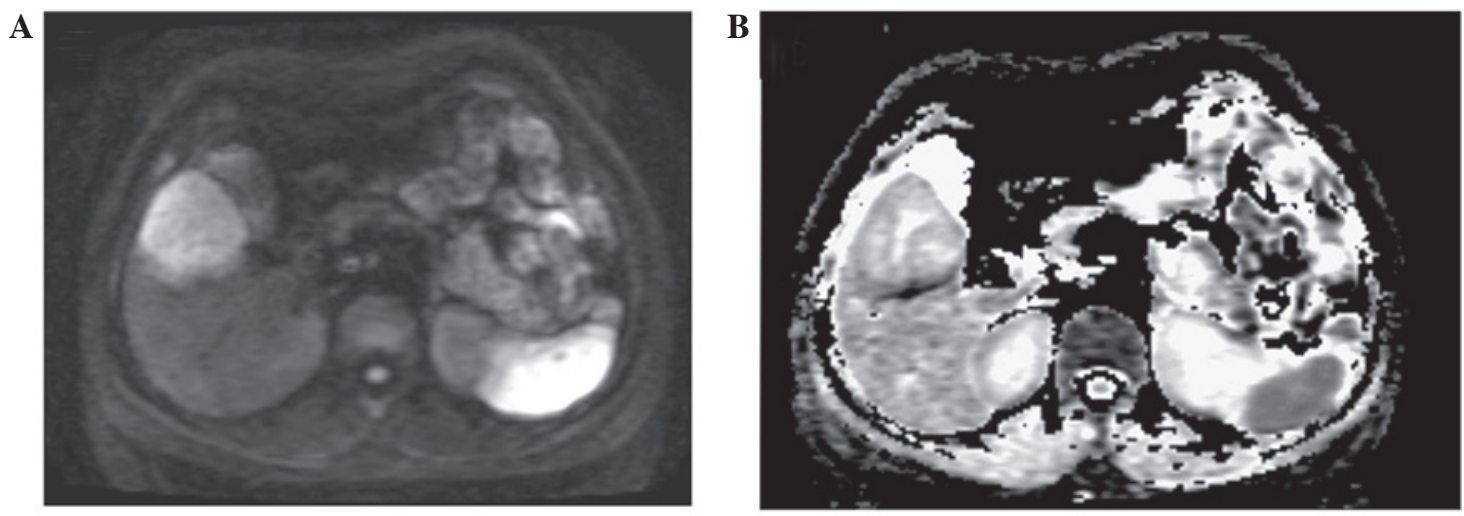

Figure 3. (A) Diffusion-weighted (DW) images showing the lesion with high signal intensity (b, $500 \mathrm{sec} / \mathrm{mm}^{2}$ ). (B) The apparent diffusion coefficient image generated from a set of DW images showing increased signal intensity in the mass. The ADC value was $1.99 \times 10^{-3} \mathrm{~mm}^{2} / \mathrm{sec}$.

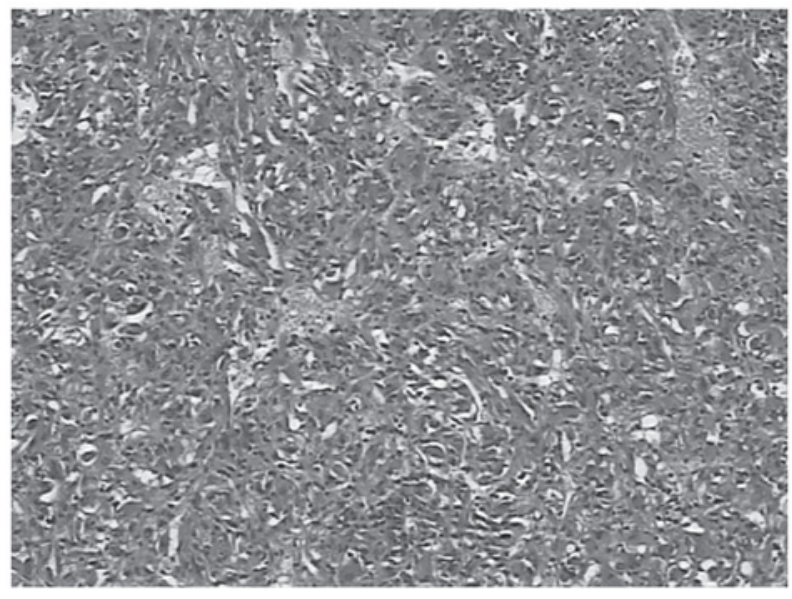

Figure 4. Pathological results showing oval or epithelioid-like tumor cells, with abundant cytoplasm, stained in red; vascular proliferation and a small amount of fat cells were also visible (hematoxylin and eosin staining; magnification, $\mathrm{x} 100)$

During the surgery, it was found that the tumor was soft with a clear margin from the surrounding, and the tumor was delicate yellowish brown at the section, without any marked abnormality in the surrounding liver parenchyma. The right lobe of the liver was resected, and a pathological examination revealed oval or epithelioid-like tumor cells, with abundant cytoplasm. Vascular proliferation and a small amount of fat cells were also found within the tumor (Fig. 4). The immunohistochemical examination indicated that the tumor was positive for human melanoma black 45 (HMB45), smooth muscle actin (SMA), cluster of differentiation (CD)31 and CD34 staining, and negative for AFP, pan-cytokeratin and S100. On the basis of these findings, the patient was diagnosed with an EMAL of the liver. The patient was healthy and free from recurrence at follow-up MRI scans, which were performed at 6 months and 1 year post-surgery.

\section{Discussion}

Hepatic EAML is a rare variant of AML that is formed almost entirely from epithelioid cells, with a proliferation of abnormal blood vessels and fewer or no lipocytes (8). Hepatic EAML commonly occurs in women in the right lobe of the liver. Patients with hepatic EAML usually have no specific clinical manifestations or physical signs, although with an increase in tumor size, certain patients may exhibit symptoms caused by tumor compression; however, results of all the laboratory examinations are usually negative. The diagnosis of EAML depends on the immunohistochemical examination (9), and the characteristic immunophenotype is dual positive for the expression of melanoma cells and smooth muscle cells, while the epithelial cell marker is negative. HMB45, one of the melanocytic markers, is the most sensitive marker, and SMA is the major marker for smooth muscle cells.

Epithelioid AML has uncertain malignant potential, and there have been rare cases of tumor recurrence, vascular invasion and metastases (10-12). Surgical resection should be considered for all symptomatic patients, who should be followed up after surgery.

The studies that previously described MRI findings (13-15) reported that hepatic EAML commonly occurred as a solitary lesion, which was hypointense on T1WI and hyperintense on T2WI. The tumor was reported as moderately or markedly enhanced, but with heterogeneous signal intensity by Gd-DTPA dynamic scanning at the arterial phase (13-15), and the signal intensity was higher or at least no less than the surrounding liver parenchyma at the portal venous phase, although the signal intensity was relatively reduced compared with the signal intensity at the arterial phase. In general, the Gd-DTPA dynamic scanning shows the feature of a 'quick wash-in and slow wash-out'. In addition, the image of the punctiform or filiform vessels could be observed in the tumor, and the pseudocapsule was visible as well. The Gd-EOB-DTPA-enhanced three-phase scan used in the current study was similar to that of the Gd-DTPA-enhanced scan reported in the literature, as Gd-EOB-DTPA shows a vascular-interstitial distribution in the first minutes after bolus injection, thus enabling a standard dynamic study of the liver (3-5). This agent is also known as gadoxetic acid disodium, and is a hepatocyte-specific contrast agent that the urinary and biliary systems eliminate in equal quantities. As a consequence of hepatocyte uptake, normal liver areas exhibit T1 shortening, whereas focal liver lesions without hepatocytes do not. Gd-EOB-DTPA has been shown to be useful in the detection of focal malignant liver lesions, including hepatocellular carcinoma and metastasis $(16,17)$. In the present 
patient, hepatic EAML showed homogeneous and significantly lower signal intensity at the hepatobiliary phase at $30 \mathrm{~min}$ post-Gd-EOB-DTPA injection compared with the normal liver parenchyma. This may be due to the fact that EAML is mainly composed of diffused epithelioid cells with diverse morphologies, containing an abundant sinusoidal vascular network, but lacking normal liver cells; therefore, the rate of the uptake of Gd-EOB-DTPA is lower $(8,9)$.

The hepatic EAML in the current study showed a high signal intensity on DWI scans, with a higher ADC value than that of the surrounding normal liver parenchyma. The main reason for this was that the tumor consisted of proliferated epithelioid cells with abundant cytoplasm and a lack of adipose tissue, which facilitated the diffusion of water molecules within the tumor leading to the high ADC value. In addition, EAML is a hypervascular lesion composed of abundant sinusoid-like vessel networks and segmentations in various sizes, which lead to increased microperfusion and an increased ADC value $(13,14)$.

The diagnosis of hepatic EAML must be differentiated from that of hepatocellular carcinoma, focal nodular hyperplasia (FNH) and hepatocellular adenoma, which are rich in blood vessels. Hepatocellular carcinoma is usually associated with a medical history of hepatitis B virus infection and cirrhosis, with an increased serum AFP level; and the dynamic MRI scan demonstrates the feature of a 'quick wash-in and quick wash-out'. Certain well-differentiated hepatocellular carcinoma cells maintain their ability to uptake Gd-EOB-DTPA and present with relatively high intensity in the liver-specific phase, while most hepatocellular carcinoma cells show low intensity during the liver-specific phase (5). The diffusion rate is limited in hepatocellular carcinoma on DWI scans; therefore, the ADC value is lower than that of the normal liver parenchyma $(6,7,16)$. The signal intensity of an MRI scan for FNH is close to that of the normal liver parenchyma, with star-like scars in the middle of the lesion; it is characterized by a delayed enhanced signal intensity during the dynamic enhanced MRI scan. FNH could take up Gd-EOB-DTPA and induce an equivalent or higher signal intensity compared with that of the normal liver parenchyma at the hepatobiliary phase (17). In addition, a higher signal intensity on the DWI scan and a higher ADC value is also observed for FNH compared with the normal liver parenchyma $(6,7,18)$. Hepatic adenoma often occurs in young and middle-aged women, who usually have a long medical history of oral contraceptive use. The lesion is often accompanied by an internal hemorrhage with degeneration of the adipose tissue, and it is usually encapsulated. The MRI signal intensity of hepatic adenoma is not homogeneous but has a clear boundary from the surrounding tissues; the homogeneous and persistent enhancement could be visualized during the enhanced scanning. However, hepatic adenoma exhibits no vascular malformation within the tumor on MRI scan. On Gd-EOB-DTPA-enhanced hepatobiliary phase images, hepatic adenoma typically appears as hypointense due to a lack of biliary canaliculi (19), while it has an equivalent or higher signal intensity on DWI scan and a mildly higher ADC value than that of the normal liver parenchyma $(6,7)$.

In summary, the present study indicated that hepatic EAML demonstrates specific features on dynamic Gd-EOB-DTPA-enhanced and DWI scans, which could be used for the differential diagnosis with other hypervascular hepatic tumors.

\section{References}

1. Xu PJ, Shan Y, Yan FH, Ji Y, Ding Y and Zhou ML: Epithelioid angiomyolipoma of the liver: Cross-sectional imaging findings of 10 immunohistochemically-verified cases. World $\mathbf{J}$ Gastroenterol 15: 4576-4581, 2009.

2. Lane BR, Aydin H, Danforth TL, Zhou M, Remer EM, Novick AC and Campbell SC: Clinical correlates of renal angiomyolipoma subtypes in 209 patients: Classic, fat poor, tuberous sclerosis associated and epithelioid. J Urol 180: 836-843, 2008.

3. Schuhmann-Giampieri G, Schmitt-Willich H, Press WR, Negishi C, Weinmann HJ and Speck U: Preclinical evaluation of Gd-EOB-DTPA as a contrast agent in MR imaging of the hepatobiliary system. Radiology 183: 59-64, 1992.

4. Motosugi U, Ichikawa T, Sou H, Sano K, Tominaga L, Muhi A and Araki T: Distinguishing hypervascular pseudolesions of the liver from hypervascular hepatocellular carcinomas with gadoxetic acid-enhanced MR imaging. Radiology 256: 151-158, 2010.

5. Ahn SS, Kim MJ, Lim JS, Hong HS, Chung YE and Choi JY: Added value of gadoxetic acid-enhanced hepatobiliary phase MR imaging in the diagnosis of hepatocellular carcinoma. Radiology 255: 459-466, 2010.

6. Miller FH, Hammond N, Siddiqi AJ, Shroff S, Khatri G, Wang Y, Merrick LB and Nikolaidis P: Utility of diffusion-weighted MRI in distinguishing benign and malignant hepatic lesions. J Magn Reson Imaging 32: 138-147, 2010.

7. Parikh T, Drew SJ, Lee VS, Wong S, Hecht EM, Babb JS and Taouli B: Focal liver lesion detection and characterization with diffusion-weighted MR imaging: Comparison with standard breath-hold T2-weighted imaging. Radiology 246: 812-822, 2008.

8. Tsui WM, Colombari R, Portmann BC, Bonetti F, Thung SN, Ferrell LD, Nakanuma Y, Snover DC, Bioulac-Sage P and Dhillon AP: Hepatic angiomyolipoma: A clinicopathologic study of 30 cases and delineation of unusual morphologic variants. Am J Surg Pathol 23: 34-48, 1999.

9. Ren N, Qin LX, Tang ZY, Wu ZQ and Fan J: Diagnosis and treatment of hepatic angiomyolipoma in 26 cases. World $\mathrm{J}$ Gastroenterol 9: 1856-1858, 2003.

10. Nguyen TT, Gorman B, Shields D and Goodman Z: Malignant hepatic angiomyolipoma: Report of a case and review of literature. Am J Surg Pathol 32: 793-798, 2008.

11. Graziano A, Santangelo M and Umana DS: Clinical evaluation of epithelioid angiomyolipoma. Ann Ital Chir 79: 135-138, 2008.

12. Xie L, Jessurun J, Manivel JC and Pambuccian SE: Hepatic epithelioid angiomyolipoma with trabecular growth pattern: A mimic of hepatocellular carcinoma on fine needle aspiration cytology. Diagn Cytopathol 40: 639-650, 2012.

13. Agaimy A, Vassos N, Croner RS, Strobel D and Lell M: Hepatic angiomyolipoma: A series of six cases with emphasis on pathological-radiological correlations and unusual variants diagnosed by core needle biopsy. Int J Clin Exp Pathol 5: 512-521, 2012.

14. Ji JS, Lu CY, Wang ZF, Xu M and Song JJ: Epithelioid angiomyolipoma of the liver: CT and MRI features. Abdom Imaging 38: 309-314, 2013.

15. Zhao Y, Ouyang H, Wang X, Ye F and Liang J: MRI manifestations of liver epithelioid and nonepithelioid angiomyolipoma. J Magn Reson Imaging 39: 1502-1508, 2014.

16. Xu PJ, Yan FH, Wang JH, Shan Y, Ji Y and Chen CZ: Contribution of diffusion-weighted magnetic resonance imaging in the characterization of hepatocellular carcinomas and dysplastic nodules in cirrhotic liver. J Comput Assist Tomogr 34: 506-512, 2010.

17. Zech CJ, Grazioli L, Breuer J, Reiser MF and Schoenberg SO: Diagnostic performance and description of morphological features of focal nodular hyperplasia in Gd-EOB-DTPA-enhanced liver magnetic resonance imaging: Results of a multicenter trial. Invest Radiol 43: 504-511, 2008.

18. Donati F, Boraschi P, Gigoni R, Salemi S, Falaschi F and Bartolozzi C: Focal nodular hyperplasia of the liver: Diffusion and perfusion MRI characteristics. Magn Reson Imaging 31: 10-16, 2013.

19. Grazioli L, Morana G, Kirchin MA and Schneider G: Accurate differentiation of focal nodular hyperplasia from hepatic adenoma at gadobenate dimeglumine-enhanced MR imaging: prospective study. Radiology 236: 166-177, 2005. 